\title{
Quantitative analysis of sialic acids in Chinese conventional foods by HPLC-FLD
}

\author{
Hongwei Li, Xingdan Fan \\ Department of Preventive Medicine, School of Public Health, Xiamen University, Xiamen, China; rocque@xmu.edu.cn
}

Received 15 November 2013; revised 3 January 2014; accepted 15 January 2014

Copyright (C) 2014 Hongwei Li, Xingdan Fan. This is an open access article distributed under the Creative Commons Attribution License, which permits unrestricted use, distribution, and reproduction in any medium, provided the original work is properly cited. In accordance of the Creative Commons Attribution License all Copyrights (C) 2014 are reserved for SCIRP and the owner of the intellectual property Hongwei Li, Xingdan Fan. All Copyright (C) 2014 are guarded by law and by SCIRP as a guardian.

\section{ABSTRACT}

Background: Sialic acids are a family of ninecarbon sugar compounds with carboxylic acyl derivatives. It exists in bacteria, fish, mammals and other living organisms, participates in and regulates many important life events, such as cell recognition, membrane flow, endocytosis and so on. Sialic acid is usually located in the outermost layer of the sugar part of the cell membrane and the key positions of secreted glycoconjugates (glycolipids, glycoprotein and lipopolysaccharide). Sialic acid (Sia) is an important material foundation for variety of the structure and founction of glycoconjugates. Sia has been known as nearly $\mathbf{5 0}$ members, including $\mathrm{N}$-acetylneuraminic acid (Neu5Ac), $\mathrm{N}$-glycouIylneuraminic acid (Neu5Gc) and deaminoneuraminic acid (KDN) as its core monomer. The rest of the sialic acids are derived from these three monomers. The contents of Sia in Chinese food products are unknown. Objective: To determine the contents of Sia in raw and cooked red meat, seafood, poultry and so on. Design: The following food products were purchased from a Chinese supermarket: pork, beef, lamb, salmon, cod, tuna, cow milk, cheese, butter, duck, chicken and chicken eggs. Human milk was collected from Xiamen Maternity and Child Health Care Hospital (Xiamen, China). All tissues were homogenized and hydrolyzed with $0.05 \mathrm{M}, 0.1 \mathrm{M}$ and $0.2 \mathrm{M}$ TFA for $150 \mathrm{~min}$ at $80^{\circ} \mathrm{C}$ in dark, respectively. The concentrations of Neu5Ac, Neu5Gc and KDN were determined by using HPLC with fluorescence detector. Results: The contents of total Sia ( $\mu \mathrm{g} / \mathrm{g}$ tissue or $\mu \mathrm{g} / \mathrm{mL}$ liquid sample) in Chinese raw meat were highest in lamb (269.60), followed by pork (254.88), duck (200.63), chicken
(162.86) and beef (88.03). The percentages of Neu5Gc were $36.08 \%, 26.48 \%, 0 \%, 0 \%$ and $28.40 \%$, respectively. Cod contained higher levels of Sia (171.63) than salmon (104.43) and tuna (77.98). Only Neu5Ac was 50 found in detected aquatic product. Egg yolk contained the highest level of Sia (682.04), and a higher level of Sia (390.67) was found in the egg white. Also our result showed that human milk contained extremely high level of Sia (602.55). Neu5Ac was the predominant form of Sia in all the deteced samples. KDN was found in cow milk only among the samples, the content was $1.14 \mu \mathrm{g} / \mathrm{g}$. Conclusion: The highest content of Sia in examined Chinese foods was found in 56 eggs, followed by lamb, pork, duck, cod, chicken, salmon, beef and tuna. Knowledge of the Sia content in conventional foods may help us to better understand possible medical disorders involving the uptake of the "non-human" Neu5Gc from our diet.

\section{KEYWORDS}

Quantitative Analysis; Sialic Acids; HPLC-FLD

\section{INTRODUCTION}

The family of sialic acids comprises approximately 50 members [1], all derivatives of the negatively charged 9carbon sugar neuraminic acid. The predominant sialic acid family found in humans is from the N-Acetylneuraminic acid (Neu5Ac, Figure 1(a)) branch. Hydroxylation of the $\mathrm{N}$-acetyl gives rise to N-glycolylneuraminic acid (Neu5Gc, Figure 1(b)), a branch present in animals including the great apes [2], yet absent in healthy humans since the gene responsible for $\mathrm{N}$-acetyl hydroxylation of Neu5Ac to Neu5Gc has been rendered 


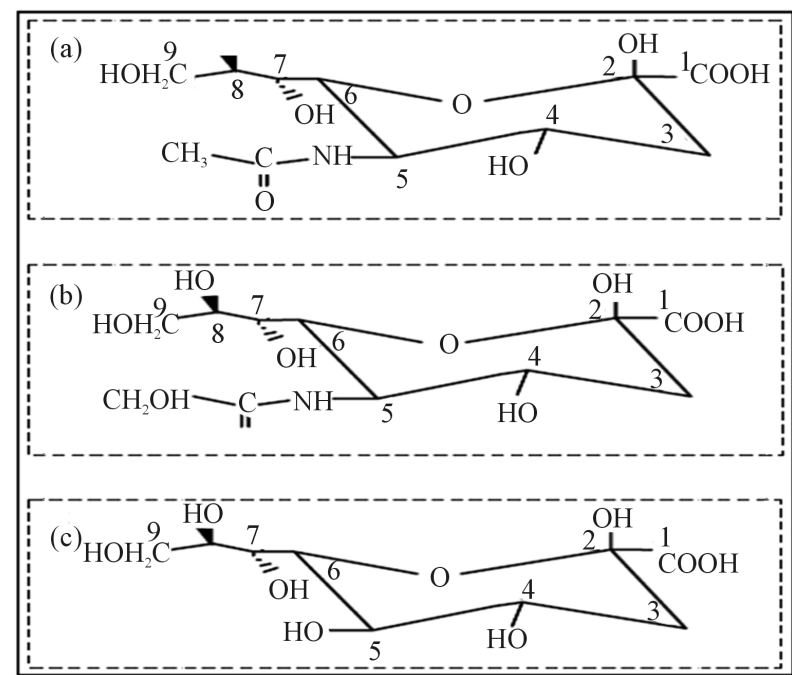

Figure 1. (a) N-acetylneuraminic acid (Neu5Ac), (b) N-glycolylneuraminic acid (Neu5Gc), (c) Ketodeoxynonulosonic acid (KDN).

inactive. The different ratio of Neu5Ac to Neu5Gc in glycans is species-specific, tissue-specific and is specific for different body fluids [3]. An unusual modification is the presence of an additional hydroxyl group instead of the amino group at position 5 of the sugar, leading to 2keto-3-deoxy-nonulosonicacid (Figure 1(c)) (keto-deoxyno-nulosonic acid, KDN) found in fish eggs (caviar) [4] and human ovarian cancer cells and ascites cells obtained [5].

Sia is found in the most vertebrate tissues and also in certain pathogenic bacteria. The mammalian central nervous system is one of the richest sources of Sia; specially brain gray matter, where Sia is mainly bound to gangliosides (65\% of total brain Sia) and glycoproteins (37\% of total Sia) [6]. Brain sialoglycopeptides have been shown to participate in the establishment of synaptic pathways, calcium transportation, binding of neurotransmitters, cell-to-cell interactions and axon regeneration $[7,8]$.

The need for Sia by the developing brain, both in utero and after birth, cannot be satisfied by anabolic metabolism alone. It is assumed that significant amounts of Sia are provided through the placenta and that human milk is provided to Sia after birth, so an exogenous source of sialic acid plays an important role in rapid brain growth. Neu5Ac is the most common form of Sia in human glycoconjugates, including glycoproteins, glycosaminoglycans, gangliosides and mucins [8], and is expressed ubiquitously throughout the human body. Neu5Gc can be found at low levels in human carcinomas, fetal tissue, and normal tissue types, e.g., endothelium and epithelium of human surgical specimens at autopsy [9]. This finding implies that Neu5Gc originates from exogenous dietary sources [10], generally red meat and milk prod- ucts [11].

The distribution of Sia is widely expressed on all mammalian tissues as either Neu5Ac, Neu5Gc, or more rarely as KDN. Animal foods, including red meat, fish and poultry, contain high-biological-value protein and important micronutrients that are required to sustain adequate human health throughout life. These animal foods also remain a core food in the diet of Chinese today.

There are no data about the contents of Sia in Chinese conventional foods until now, thus we analyzed them in raw and cooked red meet, seafood and poultry by using an established HPLC-FLD method recently.

\section{MATERIALS AND METHODS}

\subsection{Chemicals and Reagents}

It is essential to use high-quality water of high resistivity (18 $\mathrm{M} \Omega$ or better) that contains as little dissolved carbon dioxide as possible. Biological contamination should be absent. Neu5Ac, Neu5Gc and KDN from Sigma (St. Louis, MO, USA) were used as standards for identification and quantitation. 4,5-Methylenedioxy-1,2phenylenediamine dihydrochloride (DMB) was also from Sigma. Methanol and acetonitrile (HPLC grade) were from SK Chemical (Seoul, Korea). Analytically pure trifluoroacetic acid (TFA), glacial acetic acid, sodium sulfite, sodium hyposulfite, 2-mercaptoethanol were from Sinopharm Chemical Reagent Co., Ltd. (Shanghai, China). $0.45 \mu \mathrm{m}$ microporous membranes were from Shanghai Chu-Ding Analytic Instrument Limited Company (Shanghai, China).

\subsection{DMB Reagent}

$8 \mathrm{mM}$ DMB, 1.5 M glacial acetic acid, $0.25 \mathrm{M}$ sodium hyposulfite, $0.25 \mathrm{M}$ sodium sulfite, $0.8 \mathrm{mM}$ 2-mercaptoethanol.

\section{Samples and Preparations}

Food samples (beef, pork, lamb, chicken, duck, cow milk, cheese, egg, butter, ham, salmon, cod, tuna, corn and so on) were purchased in supermarket (Xiamen, China). Human milk was collected from Xiamen Maternity and Child Health Care Hospital (Xiamen, China).

\section{Preparation of Raw Food Samples}

$1 \mathrm{~g}$ of edible part of food was weighed and homogenated with pure water and metered volume at $50 \mathrm{~mL}$, liquid samples were diluted 8 times. $1 \mathrm{~mL}$ homogenated or diluent samples were hydrolyzed by $0.05 \mathrm{M}$ TFA or $0.1 \mathrm{M}$ TFA or $0.2 \mathrm{M} \mathrm{TFA}$ at $80^{\circ} \mathrm{C}$ for $1.5 \mathrm{~h}$ (according to food's distinct). Once cooled down to room temperature, hydrolyzed samples were filtered through $0.45 \mu \mathrm{m}$ microporous membrane, derived with DMB as followed. Briefly, sample $(90 \mu \mathrm{L})$ was mixed with DMB $(10 \mu \mathrm{L})$ reagent and then kept for $2.5 \mathrm{~h}$ at $50^{\circ} \mathrm{C}$ in dark. Use $10 \mu \mathrm{l}$ of the reaction mixture for HPLC analysis. 


\section{Preparation of Cooked Food Samples}

Edible part of food were weighed and recorded. Animal products were richly wraped up by aluminium-foil paper and baked at $200^{\circ} \mathrm{C}$ for $20 \mathrm{~min}$; egg and corn were boiled for $30 \mathrm{~min}$. Cooked samples were weighed and recorded for calculating the ratio of raw to cooked. Then followed the methods of raw food samples treatment.

\section{Preparation of Mixed Standards}

Different samples were hydrolyzed by different volume of TFA, mixed standards of Neu5Ac, Neu5Gc and KDN were prepared in parallel to samples. Triplet of mixture of Neu5Ac, Neu5Gc and KDN (1.0 mM each) were subjected to acid hydrolysis by isopyknic $0.2 \mathrm{M}$ TFA, 0.1 M TFA and 0.05 M TFA under the same conditions as described for samples, respectively. Use the resulting mixture $(0.5 \mathrm{mM}$ each) for linearity, coefficient of recovery and limit of quantitation studies.

\subsection{HPLC Separation of DMB Derivatives}

HPLC system from Agilent (Santa Clara, USA) was used: an Agilent 1200 HPLC system equipped with a fluorescence detector, an autosampler coupled to a HPLC pump. Separation was carried out on a LiChrosorb RP-18chromatographic column $(250 \mathrm{~mm} \times 4 \mathrm{~mm}, 5 \mu \mathrm{m})$ that was from Merck KGaA (Darmstadt, German). DMB derivatives of Sia were isocratically eluted using $7 \%(\mathrm{v} / \mathrm{v})$ methanol, $8 \%(\mathrm{v} / \mathrm{v})$ acetonitrile in high-quality water for $15 \mathrm{~min}$ at a flow rate of $0.9 \mathrm{ml} / \mathrm{min}$. All injections were performed at $30^{\circ} \mathrm{C}$. The eluant was monitored for fluorescence at $373 \mathrm{~nm}$ (excitation wavelength) and $448 \mathrm{~nm}$ (emission wavelength).

To ascertain the linearity and minimum detection limits of Neu5Ac, Neu5Gc and KDN, 3 injections $(10 \mu \mathrm{L})$ of each of the dilutions were made (Table 1). Plot peak area against injection volume to determine linearity.

All samples were double treated under same condition,

Table 1. Data for preparing standard curve of sialic acid.

\begin{tabular}{cccc}
\hline $\begin{array}{c}\text { Concentration of } \\
\text { standars in injections } \\
(\mu \mathrm{mol} / \mathrm{L})\end{array}$ & $\begin{array}{c}\text { Volume of mixture } \\
\text { standards }(0.1 \mathrm{mM} \& \\
1 \mathrm{mM} \text { each })(\mu \mathrm{L})\end{array}$ & $\begin{array}{c}\text { Volume of } \\
\text { DMB reagen } \\
(\mu \mathrm{L})\end{array}$ & $\begin{array}{c}\text { Volume } \\
\text { of water } \\
(\mu \mathrm{L})\end{array}$ \\
\hline 0.5 & 2 & 10 & 88 \\
1.0 & 4 & 10 & 86 \\
1.5 & 6 & 10 & 84 \\
2.0 & 8 & 10 & 82 \\
2.5 & 10 & 10 & 80 \\
5.0 & 20 & 10 & 70 \\
7.5 & 30 & 10 & 60 \\
10.0 & 40 & 10 & 50 \\
12.5 & 50 & 10 & 40 \\
\hline
\end{tabular}

calculate average content of two treatments (CV is less than $10 \%)$.

\subsection{Reproducibility}

To determine reproducibility, $90 \mu \mathrm{l}$ (0.9 picomole each) of the standard mixture prepared for reproducibility studies was analyzed repetitively 10 injections during $10 \mathrm{~h}$ after derivatization. Calculate peak area RSDs of 10 injections.

\subsection{Recovery Rate}

Add different concentrations of identical volume mixed standards to samples, according the above method to determine the total quantity of Neu5Ac, Neu5Gc and KDN and calculate recovery rate.

\subsection{Minimum Detection Limits (MDL)}

Defined amount of standards Neu5Ac, Neu5Gc, KDN were separately multiple diluted by water, and $90 \mu \mathrm{L}$ total dilution was derived at same condition and get different Signal-to-Noise by HPLC. According to the following formula: $\mathrm{MDL}=3 \mathrm{NW} / \mathrm{A}$ (W: concentration of object; N: the peak height of noise; A: the peak height of this concentration of Sialic acids), so when Signal-toNoise $(\mathrm{A} / \mathrm{N})$ is 3 , this concentration is the limit of quantification.

\section{RESULTS}

\subsection{Separation of Sia}

As can be seen in Figure 2, Neu5Ac, Neu5Gc and KDN are well resolved with this method. The retention times of KDN, Neu5Gc and Neu5Ac are $6.961 \mathrm{~min}$, $7.586 \mathrm{~min}$ and $9.669 \mathrm{~min}$, respectively.

Linearity of Sia Analysis with HPAE-FLD Table 2 shows that Neu5Ac, Neu5Gc and KDN have good linearity between $1 \mu \mathrm{mol} / \mathrm{L}$ to $200 \mu \mathrm{mol} / \mathrm{L}$ injections under different the hydrolysis conditions.

\subsection{Minimum Detection Limits}

According to the MDL calculation, the MDLs of Neu5Ac, Neu5Gc and KDN are $0.05 \mu \mathrm{mol} / \mathrm{L}, 0.06 \mu \mathrm{mol} /$ $\mathrm{L}$ and $0.05 \mu \mathrm{mol} / \mathrm{L}$, respectively.

\subsection{Reproducibility}

According to above method about determining reproducibility, RSD is $5 \%$, which stand for standard after derivativing is stable in $10 \mathrm{~h}$.

\subsection{Recovery Rate}

According the above method the recovery rate of 


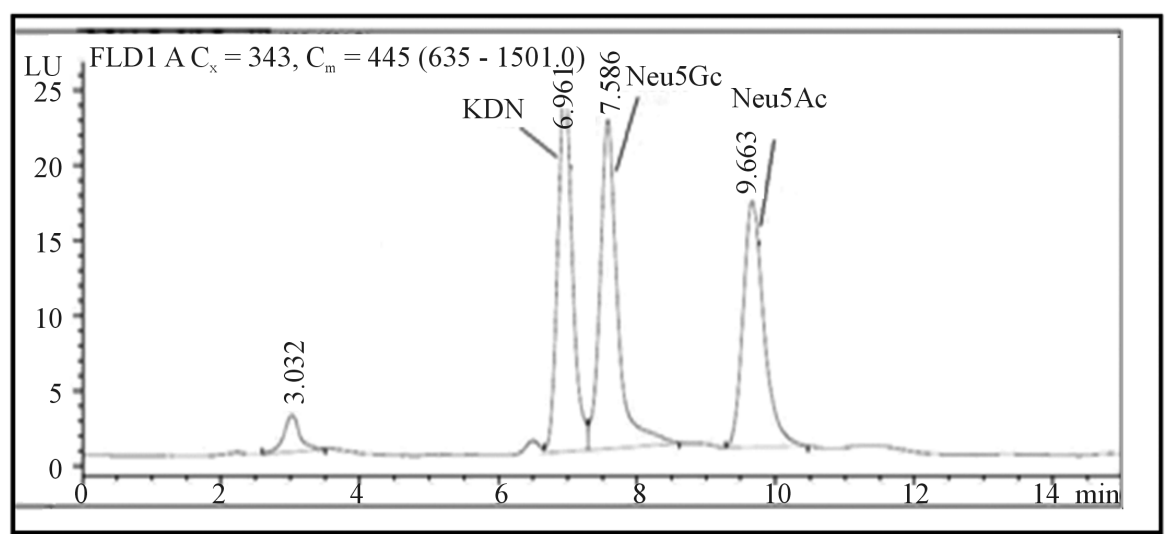

Figure 2. Chromatogram of Neu5Ac, Neu5Gc and KDN.

Table 2. Linear regression equation and correlation coefficient (r2) of Neu5Ac, Neu5Gc, and KDN.

\begin{tabular}{cccc}
\hline Concentrations of TFA to hydrolyze $(\mathrm{M})$ & Neu5Ac & Neu5Gc & KDN \\
\hline 0.05 & $\mathrm{Y}=0.0181 \mathrm{X}+4.9593 \mathrm{r}^{2}=0.9927$ & $\mathrm{Y}=0.018 \mathrm{X}+3.2900 \mathrm{r}^{2}=0.9922$ & $\mathrm{Y}=0.0208 \mathrm{X}+3.2501 \mathrm{r}^{2}=0.9937$ \\
0.1 & $\mathrm{Y}=0.0214 \mathrm{X}+0.6806 \mathrm{r}^{2}=0.9940$ & $\mathrm{Y}=0.0211 \mathrm{X}+0.8134 \mathrm{r}^{2}=0.9956$ & $\mathrm{Y}=0.0241 \mathrm{X}-1.8903 \mathrm{r}^{2}=0.9940$ \\
0.2 & $\mathrm{Y}=0.0255 \mathrm{X}-4.7169 \mathrm{r}^{2}=0.9931$ & $\mathrm{Y}=0.0258 \mathrm{X}-6.2973 \mathrm{r}^{2}=0.9937$ & $\mathrm{Y}=0.021 \mathrm{X}+0.3492 \mathrm{r}^{2}=0.9959$ \\
\hline
\end{tabular}

Neu5Ac, Neu5Gc and KDN are showed in Table 3.

\subsection{Content Analysis of Sia in Samples}

Once the method had been fully optimized, we applied it to assay food samples. The results of total Sia in Chinese conventional foods are summarized in Table 4 . The contents of total Sia ( $\mu \mathrm{g} / \mathrm{g}$ wet tissue) in Chinese raw meat was highest in lamb, followed by pork, duck, chicken and beef. The percentages of Neu5Gc were $32.30 \%$, $26.48 \%, 0 \%, 0 \%$ and $36.08 \%$, respectively. Cod contained higher levels of Sia than salmon and tuna. Only Neu5Ac was found in detected aquatic product. Egg yolk contained the highest level of Sia, and a higher level of Sia was found in the egg white. Also our result showed that human milk contained extremely high level of Sia. Neu5Ac was the predominant form of Sia in all the detected samples. KDN was found in cow milk only among the samples, the content is $1.14 \mu \mathrm{g} / \mathrm{g}$ (did not shown in Table 4).

Futhermore, cooked samples were detected. The results of total Sia ( $\mu \mathrm{g} / \mathrm{g}$ cooked tissue) are summarized in Table 5.

There are little losses of Sia in cooked samples compared to raw. The survival rates are in a range of 59.6\% to $96.6 \%$ after proofreading by ratio of raw to cooked (Table 6). There are less losses of Sia in low water content and boiled samples.

\section{DISCUSSION}

To our knowledge, sialic acids have been determined
Table 3. Average recovery rate of Neu5Ac, Neu5Gc, and KDN.

\begin{tabular}{cc}
\hline Standard & Average recovery rate \\
\hline Neu5Ac & $95-100$ \\
Neu5Gc & $96-99$ \\
KDN & $95-100$ \\
\hline
\end{tabular}

only by colorimetric assays [12,13] which are less specific and less sensitive than HPLC owing to the interference of other sugars that undergo Maillard reactions. Sia has no fluorescence in itself, but it can be produced derivatively with DMB, which can be excited by $343 \mathrm{~nm}$ exciting light and produce recognized and quantitative strong fluorescent signal without other interferences in HPLC [14]. We made a little bit to modify for component of DMB derived liquid, because we found that 0.25 $\mathrm{M}$ sodium thiosulfate and $0.25 \mathrm{M}$ sodium sulfite were used instead of 0.25 M sodium hydrosulfite [15] for DMB reagent, which enhanced the stability of derived Sia.

This quantitative assay relies on the release of sialic acid from food. Total single Sia must also be released by hydrolyzing before the DMB derivatization reaction is possible. We use TFA hydrolysis to accomplish all of this. Sia cannot bear the higher concentration of TFA used to release. To optimize hydrolysis procedure, six different concentrations of TFA to hydrolysis ranging from $0.05 \mathrm{M}$ to $0.5 \mathrm{M}$ for one sample were tested. According to peak area, best condition of hydrolysis for this sample was chosen.

Human milk is a rich source of Sia, and the content of 
Table 4. Contents of total sialic acids in conventional foods ( $\mu \mathrm{g} / \mathrm{g}$ or $\mu \mathrm{g} / \mathrm{ml})$.

\begin{tabular}{ccccc}
\hline Raw food sample & Neu5Ac & Neu5Gc & Total & $\begin{array}{c}\text { Neu5Gc, } \\
\% \text { of total }\end{array}$ \\
\hline Beef & 63.03 & 25.00 & 88.03 & 28.40 \\
Beef fat & 178.54 & 85.17 & 263.71 & 32.30 \\
Pork & 187.39 & 67.49 & 254.88 & 26.48 \\
Lamb & 172.33 & 97.27 & 269.60 & 36.08 \\
Ham & 134.76 & 44.35 & 179.11 & 24.76 \\
Chicken & 162.86 & & 162.86 & \\
Duck & 200.63 & & 200.63 & \\
Egg white & 390.67 & & 390.67 & \\
Egg yolk & 682.04 & & 682.04 & \\
Salmon & 104.43 & & 104.43 & \\
Cod & 171.63 & & 171.63 & \\
Tuna & 77.98 & & 77.98 & \\
Milk (2\% Fat 3\% Pr) & 93.75 & 3.51 & 97.26 & 3.61 \\
Butter & 206.87 & & 206.87 & \\
Cheese & 231.10 & 17.01 & 248.11 & 6.86 \\
Human milk & 602.55 & & 602.55 & \\
\hline
\end{tabular}

Table 5. Contents of total sialic acids in cooked foods ( $\mu \mathrm{g} / \mathrm{g}$ cooked tissue).

\begin{tabular}{ccccc}
\hline Cooked food sample & Neu5Ac & Neu5Gc & Total & Neu5Gc, \% of total \\
\hline Beef (cooked) & 109.59 & 45.97 & 155.56 & 29.55 \\
Pork (cooked) & 226.33 & 76.97 & 303.30 & 25.38 \\
Lamb (cooked) & 228.22 & 111.74 & 339.96 & 32.87 \\
Ham (cooked) & 147.75 & 45.27 & 193.02 & 23.45 \\
Chicken (cooked) & 210.56 & & 210.56 & \\
Duck (cooked) & 230.72 & & 230.72 & \\
Egg white (cooked) & 357.45 & & 357.45 & \\
Egg yolk (cooked) & 757.60 & 757.60 & \\
Salmon (cooked) & 191.73 & 191.73 & \\
Cod (cooked) & 220.77 & & 220.77 & \\
Tuna (cooked) & 94.96 & 94.96 & \\
\hline
\end{tabular}

total Sia in egg is the highest among analysed samples. Majority analysed animal samples are rich with Sia, and the principal constituent is Neu5Ac. Neu5Gc was found in a fewer samples, such as in cow milk. As known by animal experiment, single Sia ganglioside can effectively repair brain damage and remould cranial nervous system.
Table 6. Survival rate of Sia in cooked samples (\%).

\begin{tabular}{cccc}
\hline Food Sample & ratio of raw to cooked & Neu5Ac & Neu5Gc \\
\hline Beef & $1: 0.41$ & 71.2 & 75.3 \\
(cooked) & $1: 0.53$ & 64.0 & 60.4 \\
Pork & $1: 0.53$ & 70.1 & 60.8 \\
(cooked) & $1: 0.84$ & 92.0 & 85.7 \\
Lamb & $1: 0.50$ & 64.6 & \\
(cooked) & $1: 0.54$ & 64.5 & \\
Ham & $1: 1.00$ & 91.5 & \\
(cooked) & $1: 0.87$ & 96.6 & \\
Chicken & $1: 0.51$ & 93.6 & \\
(cooked) & $1: 0.46$ & 59.2 & \\
Duck & $1: 0.69$ & 81.8 & \\
\hline
\end{tabular}

The Sia plays the vital role in the synapse structure and the function formation. The exogenetic Sia can promote the brain growth and improve study memory behavior [13]. The examination of content of Sia in food has certain significance for food choice which promotes the brain growth and repairs brain damage.

An accurate and reliable method to quantify Sia content in food would be needed to properly be considered. Several methods to detect and/or quantify Sia have been described. In general, the first step of these protocols is to release the Sia moiety from the parental glycoconjugate. Both enzymatic and chemical reactions conducted under mild acidic conditions are preferred since only mild acid hydrolyses ensure the release of the majority of Sia residues while preserving the integrity of the molecules released. In many instances, there are steps to purify the resulting free Sia prior to quantification or identification of their precise molecular structures. Once purified, Sia can be determined by enzymatic reactions, immunochemical techniques, or by using lectins or viral agglutinins as molecular probes. Quantitative determination has traditionally been performed by colorimetry, and it is the basis of the commonly-used and thiobarbituric acid (TBA) test [16]. Currently, different versions of more laborious and sophisticated analytical technologies, such as high-performance liquid chromatography (HPLC), gas chromatography-mass spectrometry (GC-MS), NMR and GC-MS [17], high-performance anion-exchange chromatography-pulsed amperometric detection [18], and re-versed-phaseion-pair HPLC [19], have been reported for the analysis of nonderivatized free Sia. Although derivatization constitutes an additional step in analytical methods, in many cases, it increases the specificity as well as the intensity of the instrument signal, while reducing interferences and allowing a more accu- 
rate quantification. Derivatization methods that produce adducts in the presence of certain monosaccharides are also used for Sia, although some previous chemical modifications of the Sia structure, such as deacetylation or decarboxylation, are required. The most commonly used conjugation method that is specific for Sia uses the fluorescent compound 1,2-diamino-4,5-methylenedioxy-benzenedihydrochloride (DMB) [15]. Other derivatization methods using periodic acid/TBA [20] and o-phenylenediaminehydrochloride [21] have also been reported. In the present report, we describe the application of the DMB derivatization of Sia from different foods followed by HPLC separation of the resulting adducts. This method has been previously used in different biological sources, but the aim of the work described in this report was to assess its applicability to such complex food matrices.

The distribution of Sia is widely expressed on all mammalian tissues either as Neu5Ac, Neu5Gc or more rarely as KDN. Animal foods, including red meat, fish and poultry, contain high-biological-value protein and important micronutrients that are required to sustain adequate human health throughout life. These animal foods also remain a core food in the diet of China today. We analyzed the contents of Sia in raw and cooked red meet, seafood and poultry by using an established HPLC method. The content of total Sia in Chinese raw meat was the highest in lamb, followed by pork, duck chicken and beef. The percentages of Neu5Gc in each ranged from $0 \%$ to $46.08 \%$. Cooked lamb contained the highest percentage of Neu5Gc in the tested foods. Interestingly, both chicken egg yolk and white contained high Sia levels, predominantly as Neu5Ac. Koketsu et al. reported the distribution of Sia in the eggs of original Silky fowl and showed that the Sia content [22] in the yolk, albumin and chalaza of a single egg was similar with our data. Our own findings confirmed the previous reports that Neu5Gc was rare in poultry and fish, but common in milk products and enriched in red meats [11].

Importantly, humans lack the ability to synthesize Neu5Gc because of an exon deletion/frameshift mutation in the human CMAH gene 2 - 3 million years ago [11]. This mutation resulted in the complete loss of Neu5Gc expression in all human tissues [11]. However, existing biochemical pathways allow exogenous Neu5Gc to be metabolically incorporated into cultured human cells [23]. Consequently, Neu5Gc can be found at low levels in human carcinomas, fetal tissue, and normal tissue types, e.g., endothelium and epithelium of human surgical specimens at autopsy [9]. This finding implies that Neu5Gc originates from exogenous dietary sources, generally red meat and milk products [11]. As described above, there are some foods containing defined quantity of Neu5Gc. Knowledge of the Neu5Ac and Neu5Gc le- vels in conventional foods may help us to better understand possible medical disorders involving the uptake of the "nonhuman" Neu5Gc from our diet.

\section{ACKNOWLEDGEMENTS}

This project was financially supported by the Danone Nutrition Research Foundation to whom we are very grateful. Additional comments and suggestions were furnished by the teachers of School of Public Health in Xiamen University.

\section{REFERENCES}

[1] Paton, A.W., Varki, N.M., Paton, J.C., et al. (2009) A dietary non-human sialic acid may facilitate hemolyticuremic syndrome. Kidney International, 76, 140-144. http://dx.doi.org/10.1038/ki.2009.131

[2] Brinkman-Van der Linden, E.C., Sjoberg, E.R., Juneja, L.R., Crocker, P.R., Varki, N. and Varki, A. (2005) Loss of N-glycolylneuraminic acid in human evolution implications for sialic acid recognition by siglecs. Journal of Biological Chemistry, 275, 8633-8640.

http://dx.doi.org/10.1074/jbc.275.12.8633

[3] Schachter, H., Montreuil, J. and Jfg, V. (1997) Glycoproteins II. Elsevier, Amsterdam, New York.

[4] Varki, A. (1992) Diversity in the sialic acids. Glycobiology, 2, 25-40. http://dx.doi.org/10.1093/glycob/2.1.25

[5] Inoue, S., Lin, S.L., Chang, T., Wu, S.H., Yao, C.W., Chu, T.Y., Troy, F.A. and Inoue, Y. (1998) Identification of free deaminated sialic acid (2-keto-3-deoxy-D-glycero-D-galacto-nononic acid) in human red blood cells and its elevated expression in fetal cord red blood cells and ovarian cancer cells. Journal of Biological Chemistry, 273, 2719927204. http://dx.doi.org/10.1074/jbc.273.42.27199

[6] Wang, B. and Brand-Miller, J. (2003) The role and potential of sialic acid in human nutrition. European Journal of Clinical Nutrition, 57, 1351-1369. http://dx.doi.org/10.1038/sj.ejcn.1601704

[7] Sizzetoğlu, S. (2012) The determination of N-acetylneuraminic acid (Neu5Ac) and N-glycolyl-neuraminic acid (Neu5Gc) types of sialic acids in hematopoietic organ of the silkworm, bombyx moriL (Lepidoptera: Bombycidae). Kafkas Üniversitesi Veteriner Fakültesi Dergisi, 18, 147-150.

[8] Ghoshal, A. and Mandal, C. (2011) A perspective on the emergence of sialic acids as potent determinants affecting Leishmania biology. Molecular Biology International, 2011, 1-14.

[9] Tangvoranuntakul, P., Gagneux, P., Diaz, S., Bardor, M., Varki, N., Varki, A. and Muchmore, E. (2003) Human uptake and incorporation of an immunogenic nonhuman dietary sialic acid. Proceedings of the National Academy of Sciences of USA, 100, 12045-12050. http://dx.doi.org/10.1073/pnas.2131556100

[10] Padler-Karavani, V., Yu, H., Cao, H., et al. (2008) Diversity in specificity, abundance, and composition of antiNeu5Gc antibodies in normal humans: Potential implications for disease. Glycobiology, 18, 818-830. 
http://dx.doi.org/10.1093/glycob/cwn072

[11] Hedlund, M., Tangvoranuntakul, P., Takematsu, H., Long, J., Housley, G., Kozutsumi, Y., Suzuki, A., WynshawBoris, A., Ryan, A. and Gallo, R. (2007) N-glycolylneuraminic acid deficiency in mice: Implications for human biology and evolution. Molecular and Cellular Biology, 27, 4340.

[12] Martin, M.J., Martin-Sosa, S., Garcia-Pardo, L.A. and Hueso, P. (2001) Distribution of bovine milk sialoglycoconjugates during lactation. Journal of Dairy Science, 84, 995-1000. http://dx.doi.org/10.3168/jds.S0022-0302(01)74558-4

[13] Wang, B., Brand-Miller, J., McVeagh, P. and Petocz, P. (2001) Concentration and distribution of sialic acid in human milk and infant formulas. American Journal of Clinical Nutrition, 74, 510-515.

[14] Martin, M.J., Vazquez, E. and Rueda, R. (2007) Application of a sensitive fluorometric HPLC assay to determine the sialic acid content of infant formulas. Analytical Biochemistry, 387, 2943-2949. http://dx.doi.org/10.1007/s00216-007-1160-z

[15] Hara, S., Takemori, Y., Yamaguchi, M., Nakamura, M. and Ohkura, Y. (1987) Fluorometric high-performance liquid chromatography of N-acetyl- and N-glycolylneuraminic acids and its application to their microdetermination in human and animal sera, glycoproteins, and glycolipids. Analytical Biochemistry, 164, 138-145. http://dx.doi.org/10.1016/0003-2697(87)90377-0

[16] Warren, L. (1959) The thiobarbituric acid assay of sialic acids. Journal of Biological Chemistry, 234, 1971-1975.

[17] Zanetta, J.P., Pons, A., Iwersen, M., Mariller, C., Leroy, Y., Timmerman, P. and Schauer, R. (2001) Diversity of sialic acids revealed using gas chromatography/mass spectrometry of heptafluorobutyrate derivatives. Glycobiology, 11, 663-676.

http://dx.doi.org/10.1093/glycob/11.8.663

[18] Rohrer, J.S. (2000) Analyzing sialic acids using highperformance anion-exchange chromatography with pulsed amperometric detection. Analytical Biochemistry, 283, 3-9. http://dx.doi.org/10.1006/abio.2000.4643

[19] Siskos, P.A. and Spyridaki, M.H. (1999) Determination of sialic acids in biological fluids using reversed-phase ionpair high-performance liquid chromatography. Journal of Chromatography B: Biomedical Sciences and Applications, 724, 205-212. http://dx.doi.org/10.1016/S0378-4347(98)00543-X

[20] Powell, L.D. and Hart, G.W. (1986) Quantitation of picomole levels of $\mathrm{N}$-acetyl- and $\mathrm{N}$-glycolylneuraminic acids by a HPLC-adaptation of the thiobarbituric acid assay. Analytical Biochemistry, 157, 179-185. http://dx.doi.org/10.1016/0003-2697(86)90211-3

[21] Anumula, K.R. (1995) Rapid quantitative determination of sialic acids in glycoproteins by high-performance liquid chromatography with a sensitive fluorescence detection. Analytical Biochemistry, 230, 24-30. http://dx.doi.org/10.1006/abio.1995.1432

[22] Koketsu, M., Sakuragawa, E., Linhardt, R. and Ishihara, H. (2003) Distribution of N-acetylneuraminic acid and sialylglycan in eggs of the Silky fowl. British Poultry Science, 44, 145-148. http://dx.doi.org/10.1080/0007166031000085328

[23] Bardor, M., Nguyen, D., Diaz, S. and Varki, A. (2005) Mechanism of uptake and incorporation of the non- human sialic acid N-glycolylneuraminic acid into human cells. Journal of Biological Chemistry, 280, 4228-4237. http://dx.doi.org/10.1074/jbc.M412040200 